\title{
Treatment of Herpangina Patients with Xiyanping Injection: A Retrospective Study Based on the Hospital Electronic Medical Record System
}

\section{Hongjiao Geng}

China Academy of Traditional Chinese Medicine: China Academy of Chinese Medical Sciences https://orcid.org/0000-0001-5060-9365

Ruili Wei

China Academy of Traditional Chinese Medicine: China Academy of Chinese Medical Sciences

\section{Zhifei Wang}

China Academy of Traditional Chinese Medicine: China Academy of Chinese Medical Sciences

Yanming Xie ( $\sim$ kevinsunny888@sina.com )

China Academy of Traditional Chinese Medicine: China Academy of Chinese Medical Sciences

\section{Zhishuai Liu}

Renmin University of China

\section{Research Article}

Keywords: Herpangina, hospital information system, Xiyanping injection, real-world study, Traditional Chinese Medicine

Posted Date: January 14th, 2021

DOl: https://doi.org/10.21203/rs.3.rs-143787/v1

License: @ (i) This work is licensed under a Creative Commons Attribution 4.0 International License. Read Full License 


\section{Treatment of Herpangina Patients with Xiyanping Injection:A Retrospective}

Study Based on the Hospital Electronic Medical Record System

Hongjiao Geng ${ }^{1}$,Ruili Wei ${ }^{1}$,Zhifei Wang ${ }^{1}$,Yanming Xie ${ }^{1 *}$,Zhishuai Liu ${ }^{2}$

Correspondence to: Yanming Xie.Institute of Basic Clinical Medicine of Traditional

Chinese Medicine, China Academy of Chinese Medical Sciences, Beijing, 100700,

China.Email: ktzu2018@163.com

\section{Abstract}

Background:Although we have made tremendous medical advances in recent decades in vaccine and supportive therapies, the treatment of herpangina has not experienced such rapid advancement. Xiyanping injection(XYP) is a Chinese prescription with andrographolide as the main components.XYP shows strong inhibitory effects against enterovirus 71 virus,and existence of dose-response relationships in pharmacology studies.However, herpangina still lack specific therapeutics.The present study aimed to demonstrate the "real-world" experiences of herpangina in China using XYP therapy,to provide a basis for treatment of herpangina in children.

Methods:We analyzed data of patients ( $\leq 70$ years) diagnosed with herpangina and used XYP between October 2006 to October 2016 from the large electronic data warehouse (EDW), a multiple sources including hospital information system (HIS ) data of "tertiary grade A hospitals" in China. Statistical analyses were performed by SAS9.2 and R.25 software.We present the following article in accordance with the STROBE Statement reporting checklist.

Results:A total of 3423 patients of herpangina were retrieved for the analysis in this study.The mean age of the patient population was 2.66 years.Herpangina patients are often with multiple diagnosis, of which hand-foot-and-mouth disease(HFMD), bronchopneumonia and febrile seizure present a higher probability. Antibiotics were the most prescribed (17.61\%) except for XYP, followed by ascorbate $(10.40 \%)$.

Conclusions: This study is the first comprehensive retrospective review of herpangina for XYP that has been used in a clinical setting. The present findings of clinical characteristics are fairly consistent with previous clinical studies.Clinical 
medication use of herpangina were basically in accordance with Expert Consensus of Herpangina in China(2019).Meanwhile,there are abuse problems of antibiotics and antivirals.Despite the underlying mechanisms in antibacterial and antiviral of XYP, the exact clinical efficacy is yet to be revealed.The efficacy and safety of XYP with Western medicines has not been studied.

Keywords:Herpangina;hospital information system; Xiyanping injection; real-world study; Traditional Chinese Medicine

\section{Introduction}

Herpangina is one of the multimorbidity and

Common Diseases of the Pediatrics, which

is a special type of upper respiratory infections.Herpangina caused by enterovirus(EVs) and coxsackievirus presenting as fever,sore throat,oropharyngeal herpes and superficial ulcers[1].Most herpangina patients have mild symptoms,but some may occur serious complications, even fatal[2-3].

Xiyanping injection(XYP),with andrographolide(Fig.1)as the main components,shows strong inhibitory effects against enterovirus 71 and respiratory syncytial virus,and existence of dose-response relationships in $\begin{array}{llll}\text { pharmacology } & \text { studies[4-5].XYP the }\end{array}$ Consensus/Guideline-Recommended medication for hand-foot-and-mouth disease(HFMD) and viral pneumonia in children,et al[6],also used in the treatment of herpangina clinically.

The current status of the disease caused by enterovirus 71 infections: epidemiology, molecular epidemiology, vaccine development and pathogenesis[7-8].Although a commercial EV-A71 vaccine was available in mainland China in 2016[9],but it has 
no cross-protective immunity in other enterovirus serotypes[10].Herpangina still lack specific therapeutics[11].The present study aimed to demonstrate the "real-world" experiences of herpangina in China using XYP therapy,to provide a basis for treatment of herpangina in children.We present the following article in accordance with the STROBE Statement reporting checklist.

\section{Methods}

\section{Data Source}

This is a retrospective study based on HIS data. This analysis is based on a large electronic data warehouse (EDW), which was extracted from multiple sources including HIS data of sixty "tertiary grade A hospitals" in China (The EDW was built by the lead researcher YX, Institute of Clinical Basic Medicine of Traditional Chinese Medicine, China Academy of Chinese Medical Sciences). This is the largest EDW in China. These electronic data contain hospitalization records, hospitalization orders, diagnosis, test results, and economic indicators.

The key was engineered in the data extraction process, and the data were normalized and standardized. To overcome the heterogeneous data integration challenges of HIS data, we proposed (extract, transform, load) ETL technology. The HIS data were centralized and standardized [12].

\section{Study design}

This study was established in departments of 14 hospitals located in China.This was a retrospective, non interventional study involving secondary use of data collected from the EDW database during October 2006 to October 2016 in China.Study flow chart 
was showed in Fig. 2.

\section{Data Standardization}

Before the statistical analysis, the original HIS data must be standardized. The Western medicine diagnosis was standardized in reference to ICD-10 [13]. Western medicine was standardized according to the drug code of the Anatomical Therapeutic Chemical (ATC), and Chinese patent medicine was standardized according to China National Medical Insurance Directory.

\section{Inclusion criteria for participants}

Data were collected from patients whose discharge diagnoses were classified coded 'B00.202', referred to herpangina.Meanwhile, they were prescribed and took XYP and aged less than 70 years.

\section{Study Variables}

Study variables were characteristics of herpangina patients and the combined diagnosis, characteristics of XYP medication use,combination drugs and intercorrelations among drugs.

\section{Statistical analysis}

The data were imported into SAS 9.2 (SAS Inc., Renmin University of China version). Descriptive analysis, including numbers and percentages, was performed for every item.Associations were analyzed using the apriori function in the arules package in R.25.

\section{Results}

\section{Characteristics of patients}


A total of 3423 patients in the EDW database were retrieved for the analysis in this study.Characteristics of all patients are displayed in Tables 1.

\section{Combined diagnosis}

Combined diagnosis of patients were analyzed.The result of the analysis indicates that,except for herpangina, HFMD, bronchopneumonia and febrile seizure present a higher probability. The detailed disease distribution was illustrated in Fig.3.

\section{Characteristics of XYP medication use}

A total of 38788 medical advice records were extracted.There were 4238 XYP records in the database.Characteristics of XYP medication use are displayed in Table 2.

Set age of patients as the independent variable x.Regression analysis was performed with single doses of XYP as the dependent variable $\left(f(x)=0.10749 x^{2}+0.02776 x+3.87\right)$.Curves were drawn according to the equation.A curvilinear relationship was shown between age of patients and single doses(Fig.4).

\section{Combination drugs}

Other combination drugs were also analysed.The results are shown in the Table3.

\section{Association analysis of drugs}

In this study,we used the Apriori algorithm to analyze the association rules of XYP and combination drugs in all prescriptions. The terms confidence, support, and lift are metrics used in the rule selection to be described next.Support and confidence are measures of the interestingness of a rule.Support reflects the probability that drug A 
and drug B appear at the same time.It is used to measure of the importance of an association rule, which indicates the frequency of this rule.Confidence is to use drug $\mathrm{B}$ on the basis of drug A,which is a measure of the accuracy of association rules and indicates the strength of the rules[14].

Analyze the application of XYP combined with two or three drugs.Extract drug combinations with support greater than 10\%. The detailed values are given in Table 4 .

It is visible in table4 that the first three of support of XYP combining are(1)Mezlocillin,Penicillin(2)VitaminB6,VitaminC(3)Shuangliaohoufengsan,Vitamin C,and(1)VitaminC,Mezlocillin,Penicillin(2)VitaminB2,Mezlocillin,Penicillin(3) Vitamin B2,Vitamin B6, Vitamin C.

\section{Discussion}

\section{Clinical characteristics of herpangina}

As a common upper respiratory infectious disease in children, herpangina has strong infectivity and spread rapidly.According to previous epidemiological studies,herpangina affects children, in particular children less than 5 years old,and the male:female ratio is 1.14-1.90:1[15].In the present study,toddlers (1-3 years of age) had the highest number( $81.53 \%)$, and the ratio between male to female is 1.5:1.These conclusions are congruent with previous epidemiological reports.Symptoms of herpangina generally persist for 5-10 days, and majority of the patients 
had good prognosis. This study showed that, the clinical cure and improvement rates were as high as $99.45 \%$.

As the highly coincident between herpangina and HFMD,there were no differences between the two diseases in age, gender, nucleotide sequences and onset seasons.Therefore, the two diseases may be transformed into each other[18-19].As a result,some herpangina cases had a concurrent diagnosis of HFMD in the present study.EVs, as single-stranded positive-sense RNA viruses,mainly spread through oral-oralor oral-fecal transmission, as well as respiratory droplets.Thus, it mainly causes respiratory and gastrointestinal diseases.A clinical monitoring study done in Beijing of China reported leukocyte count increase $\left(>10 \times 10^{9} / \mathrm{L}\right)$ for $2.3 \%$ of children, high neutrophil percentage $(>70.0 \%)$ in $51.4 \%$, decreased percentage of lymphocytes $(<20.0 \%) \quad$ in $48.6 \%$ and the elevated serum C-reactive protein (CRP) levels in the children[20].It demonstrated that children with herpangina are frequently complicated by secondary bacterial infection.A growing number have been found,EVs possed the ability to invade the central nervous system and cause potentially fatal neurological symptoms including encephalitis, meningitis and paralysis[21].As reported,EVs infections were the most common cause of meningitis,encephalitis in adolescents (ages 17 and younger)[22].Of those, infants were highly susceptible population with a subsequent mortality rate as high as $10 \%[23]$. The results of the current study also demonstrated that patients had diagnosis of herpangina mostly with 
HFMD, bronchopneumonia,febrile, acute tonsillitis, acute gastroenteritis, viral encephali tis and myocardial damage, et al.

\section{Analysis of XYP medication use}

XYP is frequently used in the clinical treatment of HFMD and herpangina[24-25].Modern pharmacology research confirmed that XYP occupied binding sites in proteins nucleic acids to inhibition of viral replication.Furthermore,XYP was very effective at reducing prostaglandin synthesis at the site of inflammation.Moreover, it can increase serum level of interleukin and lysosomes,improve $\mathrm{B}$ and $\mathrm{T}$ lymphocytes density in spleen, increase neutrophils and phagocytosis phagocytic capacity,stimulate the release of adrenocorticotropic hormone (ACTH) and immunoglobulins enhancing the body's immune function[26-28].

According to the results of this study, the most commonly used dosage of XYP was less than $100 \mathrm{mg}$ one time ( $84.2 \%$ ). Single dose of an adult should be $250-500 \mathrm{mg}$, and children 5-10mg/kg,according to the specification from the seller. Because of,the data about the participants' weight were unavailable in this study, we referenced in"The WHO Child Growth Standards"[29],to explore the actual dosage of children. Standard weight was calculated based on age. The standard dose in children estimated from the dose recommended by the instructions. The therapeutic dose for children and adults were lower than the recommended dose of the instructions.

\section{Clinical medication use of herpangina}


Analysis of clinical data suggests that antibiotics and vitamins were commonly used drugs combination for the treatment of herpangina.Our analysis showed that the most prescribed antibiotics were Penicillin (ie,5.93\% of all medication use of herpangina), followed by Mezlocillin (5.17\%).And the most prescribed vitamins were Vitamin C (ie, $7.87 \%$ of all medication use of herpangina), followed by Vitamin B2 (5.18\%).Trend analysis of combined drug use identified that XYP often combined with two categories of drugs above(1) Penicillin,Mezlocillin;(2)Vitamin B6,Vitamin C;(3Vitamin C,Mezlocillin,Penicillin(4) Vitamin B2,Mezlocillin,Penicillin; (5) Vitamin B2,Vitamin B6,Vitamin C.As upper respiratory viral infections are frequently complicated by secondary bacterial infection[20], antibiotics were more often prescribed in clinical.Based on prior biological principles studies, there is increasing interest regarding the use of vitamin $\mathrm{C}$ for acute inflammatory disease prevention and therapy.Administering vitamin $\mathrm{C}$ is seen to increase generation of IFN- $\alpha$ and IFN- $\beta$, the production of pro-inflammatory cytokines and oxidative killing, lymphopoiesis proliferation and function, to modulate immune function[30-33].As a potent antioxidant, vitamin $\mathrm{C}$ is capable of scavenging oxygen radicals and other cellular antioxidant, reducing oxidative damages caused by virus[34-35]. Despite vitamin $\mathrm{C}$ may be effective on virus infections in biologically,current evidence-based options for treatment remain limited.We were unable to obtain consistent findings to support this view[36]. Riboflavin(Vitamin B2) as a coenzyme of multiple oxidoreductases of body,is a key regulator of cellular respiration, and 
involved in the oxidation of the human body[37]. The lack of riboflavin(Vitamin B2) can induce metabolic disorders in mouth,eyes, external genitalia,presenting with glossitis, cheilitis et al.Partially clinical evidence demonstrated that compared to control,the effect of riboflavin-assisted on herpangina was significant[38-39].Vitamin B6 have been found to regulate immunity and have an active effect on oral ulceration healing[40-41].Vitamin B6 may have adjuvant effects on the treatment of herpangina.The paucity of evidence on the comparative efficacy and safety of the combination of these drugs, further research is needed for validation.

Furthermore, We found some irrational use of medicines in China.For example, combined application of Penicillin and Mezlocillin was found by association analysis.As the same antibiotic, combination of penicillin and mezlocillin was outside of the clinical norms on antibiotic use.However,the resultant inference was found through association analysis which does not necessarily reflect real cases.Furthermore, as recommendations based on available expert consensus, there is currently no specific antiviral western drug for herpangina.Antiviral medications such as acriflavine,ganciclovir and vidarabine are drugs against DNA viruses,for RNA viruses are often ineffective.Thus, these drugs should not be used for treatment of herpangina[42]. There is a need to emphasize on the rational use of antimicrobials and strict adherence to the standard treatment guidelines.

\section{Conclusions}


This study is the first comprehensive retrospective review of herpangina for XYP that has been used in a clinical setting. The present findings of clinical characteristics are fairly consistent with previous clinical studies.Clinical medication use of herpangina were basically in accordance with Expert Consensus of Herpangina in China(2019).Meanwhile,there are abuse problems of antibiotics and antivirals.Despite the underlying mechanisms in antibacterial and antiviral of XYP, the exact clinical efficacy is yet to be revealed.The efficacy and safety of XYP with Western medicines has not been studied.It is important to continue investigations in this area to improve the clinical value of XYP and the clinical treatment of herpangina.As a real-world retrospective study, our results may have been affected by confounding factors.In this paper, 3423 patients applying XYP were screened.The number of records was small and there was missingness in the data.Therefore, the study was slightly underpowered.

\section{Abbreviations}

XYP:Xiyanping injection;EDW:electronic data warehouse;HIS:hospital information system;HFMD:hand-foot-and-mouth disease;ETL:extract, transform, load;ATC: Anatomical Therapeutic Chemical;CRP:C-reactive protein ;ACTH:adrenocorticotropic hormone.

\section{Acknowledgements}

We sincerely thank all the experts who have insights into this paper.

\section{Authors' contributions}

$\mathrm{X}$ YM and W ZF conceived the study and participated in its design and coordination. $\mathrm{X}$ YM and W ZF designed the schema of the HIS database and performed data acquisition at each medical center. L ZS performed the data analysis. G HJ and W RL wrote the manuscript with contributions from all other authors. All authors approved the final manuscript.

\section{Funding}

This work was supported by the Research Fund of National Key Research and Development Plan Project of China (2018YFC1707410).

\section{Availability of data and materials}


All datasets are presented in the main paper.

\section{Ethics approval and consent to participate}

This electronic data warehouse (EDW) analysis was approved by the Ethical Review Board of the Institute of Basic Research in Clinical Medicine, China Academy of Chinese Medical Sciences. Analysis was conducted using publicly available data files from hospital information system (HIS) database; Data extracted from hospital information system database do not require individual informed consent. However, a data use agreement submission was required to access the HIS Research Data File. The patient data in this study was anonymously managed in all stages, including stages of data cleaning and statistical analyses.

\section{Consent for publication}

Not applicable.

\section{Competing interests}

The authors declare that they have no competing interests.

\section{Author details}

${ }^{1}$ Institute of Basic Clinical Medicine of Traditional Chinese Medicine, China Academy of Chinese Medical Sciences, Beijing, 100700, China; ${ }^{2}$ School of Statistics, Renmin University of China, Beijing, 100872, China.

\section{References}

[1]Hu YM, Jiang ZF, Zhu FT.Practical Pediatrics [M].7th edition.Beijing:People's Medical Publishing House,2002:1167-1175.

[2]Puenpa J,Mauleekoonphairoj J,Linsuwanon P,et al.Prevalence and characterization of enterovirus infections among pediatric patients with hand foot mouth disease,herpangina and influenza like illness in Thailand,2012[J].PLoS One,2014,9(6):e98888.DOI:10.1371/journal.pone.0098888.

[3]Takechi M,Fukushima W,Nakano T,et al.Nationwide Survey of Pediatric Inpatients With Hand,Foot,and Mouth Disease,Herpangina,and Associated Complications During an Epidemic Period in Japan:Estimated Number of Hospitalized Patients and Factors Associated With Severe Cases.J Epidemiol.2019

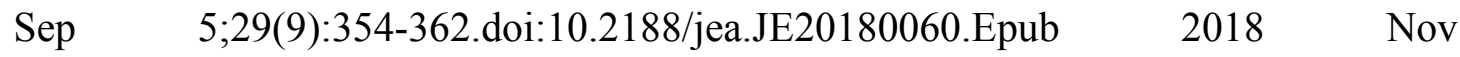




\section{PMID:30416163;PMCID:PMC6680054.}

[4]Wang SY, He M, Xie N, et al.In vitro anti-influenza pharmacodynamics study of andrographolide sulfonate[J].Central South China Pharmaceutical,2013,11(5):331.

[5]Jing J,Wang SY,Xie N, et al.In vitro anti-adenovirus pharmacodynamics study of andrographolide sulfonate[J].Chinese Journal of Experimental Formulas,2012,18(21):175.

[6]Wang ZF,Rong P,Ma R, et al.Expert consensus on the clinical application of Xiyanping injection (children's edition) [J].Chinese Journal of Chinese Materia Medica, 2019, 44(14):2932-2936.

[7]Xu SJ,He YQ,Zhang RL,et al.Near-Complete Genome Sequences of 12 Coxsackievirus Group A Strains from Hand,Foot,and Mouth Disease and Herpangina Cases with Different Clinical Symptoms.Microbiol Resour Announc.2019 Feb 21;8(8):e01655-18.

doi:10.1128/MRA.01655-18.PMID:30834371;PMCID:PMC6386572.

[8]Chang YK, Chen KH,Chen KT.Hand,foot and mouth disease and herpangina caused by enterovirus A71 infections:a review of enterovirus A71 molecular epidemiology,pathogenesis,and current vaccine development. Rev Inst Med Trop Sao $\begin{array}{lll}\text { Paulo.2018 Nov 8;60:e70.doi: } & \text { Nov }\end{array}$ 10.1590/S1678-9946201860070.PMID:30427405;PMCID:PMC6223252.

[9]Chen L,Xu SJ,Yao XJ,et al.Molecular epidemiology of enteroviruses associated with severe hand,foot and mouth disease in Shenzhen,China, 2014-2018.Arch Virol.2020 Oct;165(10):2213-2227.doi: 10.1007/s00705-020-04734-z.Epub 2020 Jul 14.PMID:32666145;PMCID:PMC7360124.

[10]Li R,Liu L,Mo Z,et al.An inactivated enterovirus 71 vaccine in healthy children.N $\begin{array}{llll}\text { Engl } & \text { J } & \text { Med.2014 } & \text { Feb }\end{array}$ 27;370(9):829-37.doi:10.1056/NEJMoa1303224.PMID:24571755.

[11] Zhao J.Observation on the efficacy of Xiyanping injection in the treatment of 40 children with herpetic angina[J].Journal of Clinical Rational Use,2019,12(11):21-22+20.

[12]Zhuang Y, Xie BT, Weng SX, et al.Discussion on the design method of HIS "real 
world" integrated data warehouse after the market of traditional Chinese medicine [J].Chinese Journal of Chinese Materia Medica, 2011, 36(20): 2880.

[13]World Health Organization.International Statistical Classification of Diseases and Related Health Problems (10th Revision) [M].Version 2. Translated by Dong Jingwu. Beijing: People's Medical Publishing House, 2008;

[14]Zhao J.Research on Association Rules Mining Algorithm [D].Taiyuan:Taiyuan University of Technology,2012.

[15]Zhao TS,Du J,Liu HY, et al.Research progress in epidemiology and etiology of herpetic angina[J].Chinese Journal of Virology,2020,10(05):385-390.

[16]Chen Y, Huang L, Zhao Y.Clinical analysis of 212 cases of herpetic angina in children[J]. China Practical Medicine,2016,11(4):71-72.

[17]Wu WJ.Clinical analysis and pathogenic characteristics of herpetic angina[D]. Wenzhou: Wenzhou Medical University, 2017.

[18]Zhou WH, Li YF, Zhang YY.Research progress on the relationship between herpetic angina and hand, foot and mouth disease[J].Prevention and Treatment of Oral Diseases,2020,28(01):61-64.

[19]Yao X,Bian LL,Lu WW,et al.Epidemiological and etiological characteristics of herpangina and hand foot mouth diseases in Jiangsu, China,2013-2014.Hum Vaccin Immunother.2017 Apr 3;13(4):823-830.doi: 10.1080/21645515.2016.1236879.Epub 2016 Oct 21.PMID:27768527;PMCID: PMC5404359.

[20]Zhao TS,Du J,Li HJ,et al.Molecular epidemiology and clinical characteristics of herpangina children in Beijing,China:a surveillance study.PeerJ.2020 Oct 15;8:e9991.doi:10.7717/peerj.9991.PMID:33088614; PMCID:PMC7568857.

[21]Majer A,McGreevy A,Booth TF.Molecular Pathogenicity of Enteroviruses $\begin{array}{llll}\text { Causing Neurological Disease.Front Apr } & \text { Microbiol.2020 A }\end{array}$ 9;11:540.doi:10.3389/fmicb.2020.00540.PMID:32328043;PMCID:PMC7161091.

[22]Hasbun R,Wootton SH,Rosenthal N,et al.Epidemiology of Meningitis and Encephalitis in Infants and Children in the United States,2011-2014. Pediatr Infect Dis J.2019 Jan;38(1):37-41.doi:10.1097/INF.0000000000002081.PMID:30531527. [23]Rhoades RE,Tabor-Godwin JM,Tsueng G,Feuer R.Enterovirus infections of the 
central nervous system. Virology.2011 Mar 15;411(2):288-305.doi: 10.1016/j.virol.2010.12.014.Epub 2011 Jan 20. PMID:21251690;PMCID: PMC3060663.

[24]Hu XJ,Li JX.The clinical efficacy of Pudilan Xiaoyan oral liquid combined with Xiyanping injection in the treatment of children with herpetic angina and its influence on inflammatory factors [J].Contemporary Medicine, 2020,26(24):10-13.

[25]Tang JS.Observation on the clinical efficacy of Xiyanping in the treatment of herpetic angina in children and its effect on peripheral blood granulocytes[J].Digest of World Latest Medical Information,2017,17(92): 84.

[26]Zhu QX, Zhu CH, Liao XS, et al. Xiyanping injection in the treatment of 27 cases of common hand, foot and mouth disease[J]. Journal of Practical Pediatrics,2012, 27(10): 780-782.

[27]Ding JB,Zhang JY, Tian YM, et al.Meta analysis of Xiyanping injection in the treatment of hand, foot and mouth disease[J].Global Chinese Medicine,2013, 6(8):585-588.

[28]Li YY.The effect of Xiyanping and ribavirin in the treatment of children with hand, foot and mouth disease[J].Chinese Continuing Medical Education,2016,8(20): 178-179.

[29]de Onis M.4.1 The WHO Child Growth Standards.World Rev Nutr Diet. 2015;113:278-94.doi:10.1159/000360352.PMID:25906897.

[30]Carr AC,Maggini S.Vitamin C and Immune Function.Nutrients.2017 Nov 3;9(11):1211.doi:10.3390/nu9111211 PMID:29099763; PMCID: PMC5707683.

[31]May JM,Harrison FE.Role of vitamin $\mathrm{C}$ in the function of the vascular endothelium.Antioxid Redox Signal.2013 Dec 10;19(17):2068-83.doi: 10.1089/ars.2013.5205.Epub 2013 May 29.PMID:23581713;PMCID: PMC3869438.

[32]Leibovitz B,Siegel BV.Ascorbic acid and the immune response.Adv Exp Med Biol.1981;135:1-25.doi:10.1007/978-1-4615-9200-6_1.PMID:7010958.

[33]Dey S,Bishayi B.Killing of S.aureus in murine peritoneal macroph ages by Ascorbic acid along with antibiotics Chloramphenicol or Ofloxacin:Correlation with inflammation.Microb Pathog.2018 
Feb;115:239-250.doi:10.1016/j.micpath.2017.12.048.Epub

PMID:29274459.

[34]Oudemans-van Straaten HM,Spoelstra-de Man AM,de Waard MC.Vitamin C revisited.Crit Care.2014 Aug 6;18(4):460.doi:10.1186/s13054-014-0460-x. PMID:25185110;PMCID:PMC4423646.

[35]May JM,Harrison FE.Role of vitamin $\mathrm{C}$ in the function of the vascular endothelium.Antioxid Redox Signal.2013 Dec 10;19(17):2068-83.doi: 10.1089/ars.2013.5205.Epub 2013 May 29.PMID:23581713;PMCID: PMC3869438. [36]Colunga Biancatelli RML,Berrill M,Marik PE.The antiviral properties of vitamin C.Expert Rev Anti Infect Ther.2020 Feb;18(2):99-101.doi: 10.1080/14787210.2020.1706483.Epub 2019 Dec 23.PMID:31852327.

[37]Chu KD.Analysis of the curative effect of riboflavin sodium phosphate injection in the treatment of 64 children with hand, foot and mouth disease[J]. Hainan Medicine, 2009, 20(5): 78-79.

[38]Chen YY.The clinical effect of Xiaoer Qingyan granule combined with riboflavin sodium phosphate and interferon in the treatment of herpetic angina[J].Clinical Medical Research and Practice,2019, 4(08):113-114.

[39]Liu XL, Qin QY.The effect of riboflavin sodium phosphate combined with interferon on CRP,IL-10 and TNF- $\alpha$ in children with herpetic angina[J].International Medicine \& Health Guidance News,2019(01):120- 123.

[40]Ueland PM,McCann A,Midttun Ø,Ulvik A.Inflammation,vitamin B6 and related pathways.Mol Aspects Med.2017 Feb;53:10-27.doi: 10.1016/j.mam.2016.08.001.Epub 2016 Sep 1.PMID:27593095.

[41]Zhou J. Combination of vitamin $\mathrm{C}$ and vitamin B6 in the treatment of hand-foot-mouth disease in children[J]. Qiqihar Medical College Journal, 2011, 32(07):1106.

[42]Infectology Group of Pediatrics Branch of Chinese Medical Association, National Center for Infectious Diseases Medical Quality Control. Expert consensus on diagnosis and treatment of herpes angina (2019 edition) [J]. Chinese Journal of Pediatrics, 2019, 57(3) :177-180. DOI:10.3760/cma.j.issn.0578-1310.2019.03.004. 
Figures

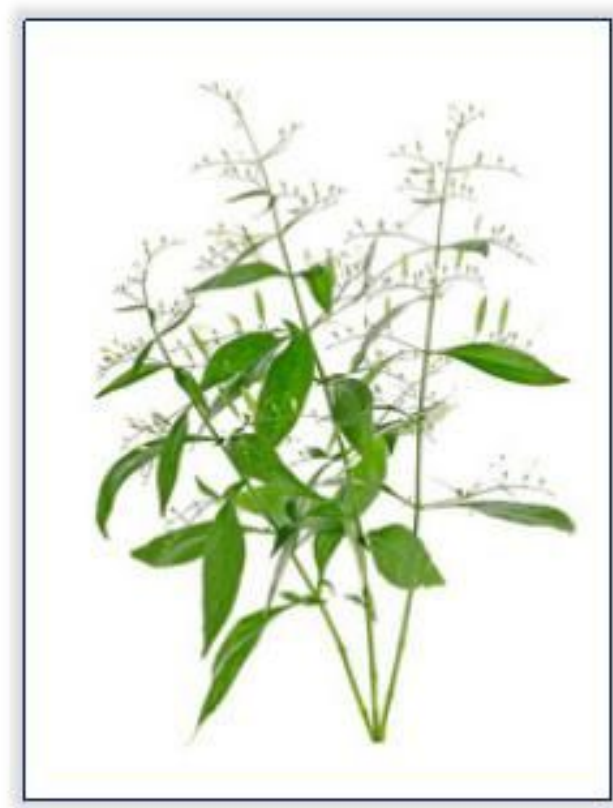

A. The whole plant

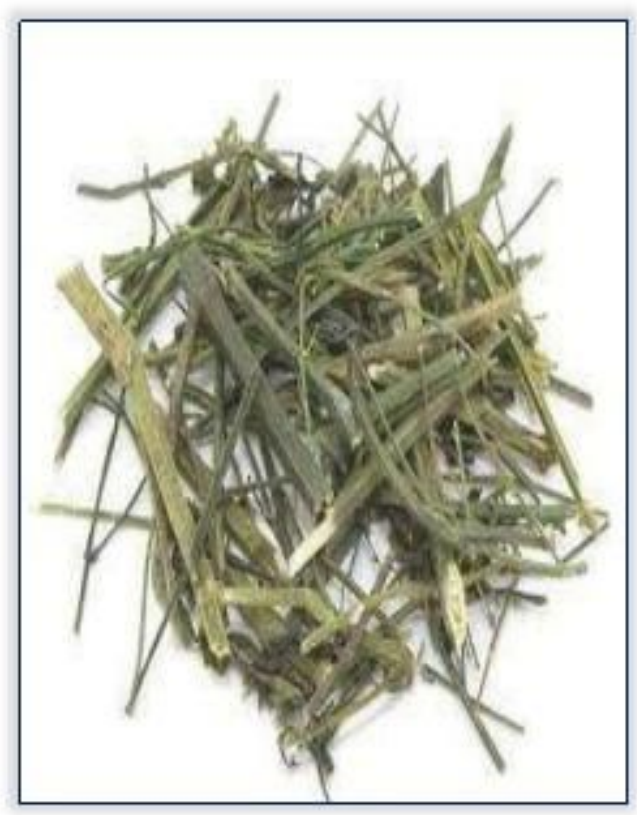

B.Chinese herbal piece for pharmaceutical use

\section{Figure 1}

Andrographis (A) The whole plant.(B) Chinese herbal piece for pharmaceutical use 


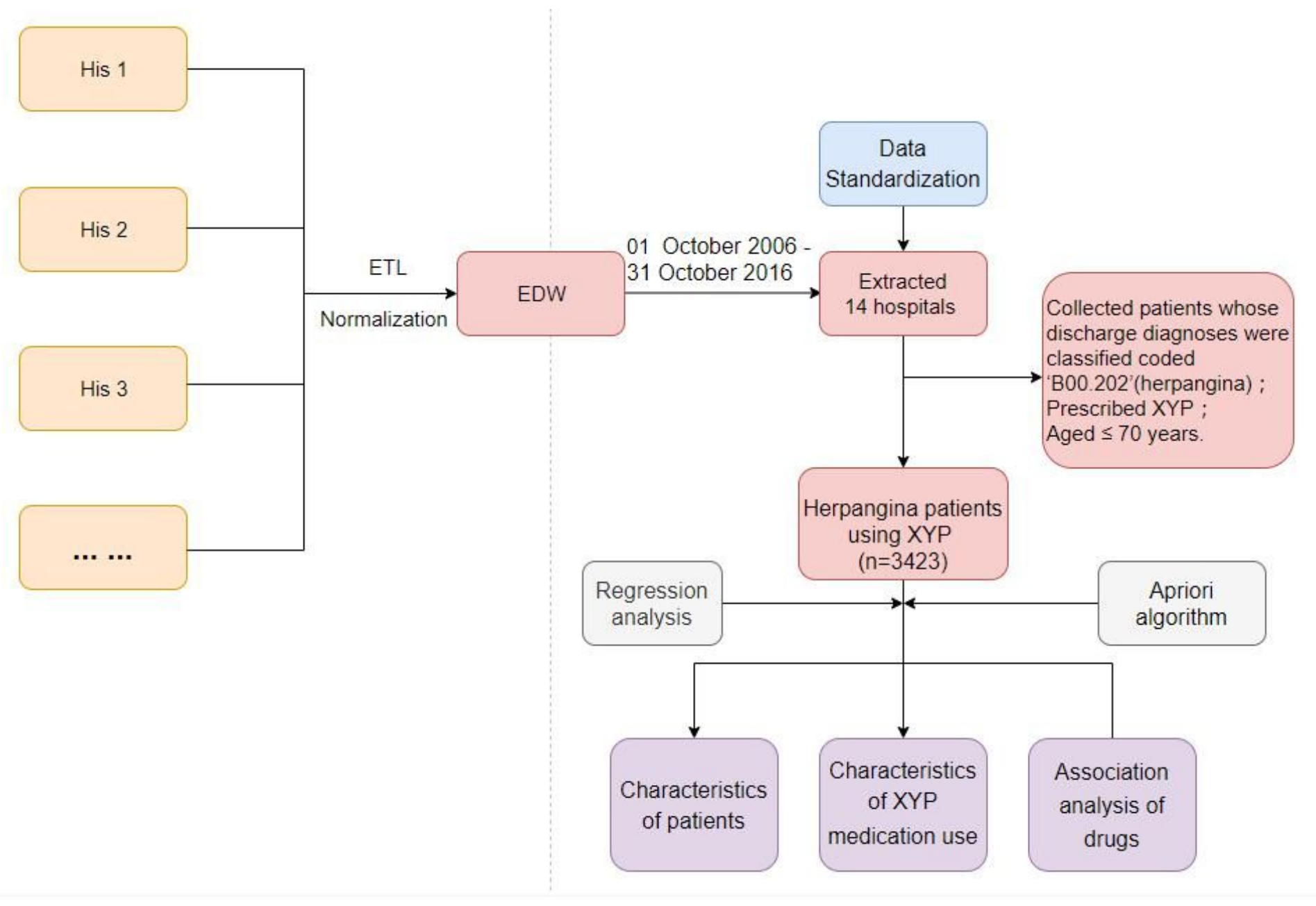

Figure 2

The main flow-chart of this study 


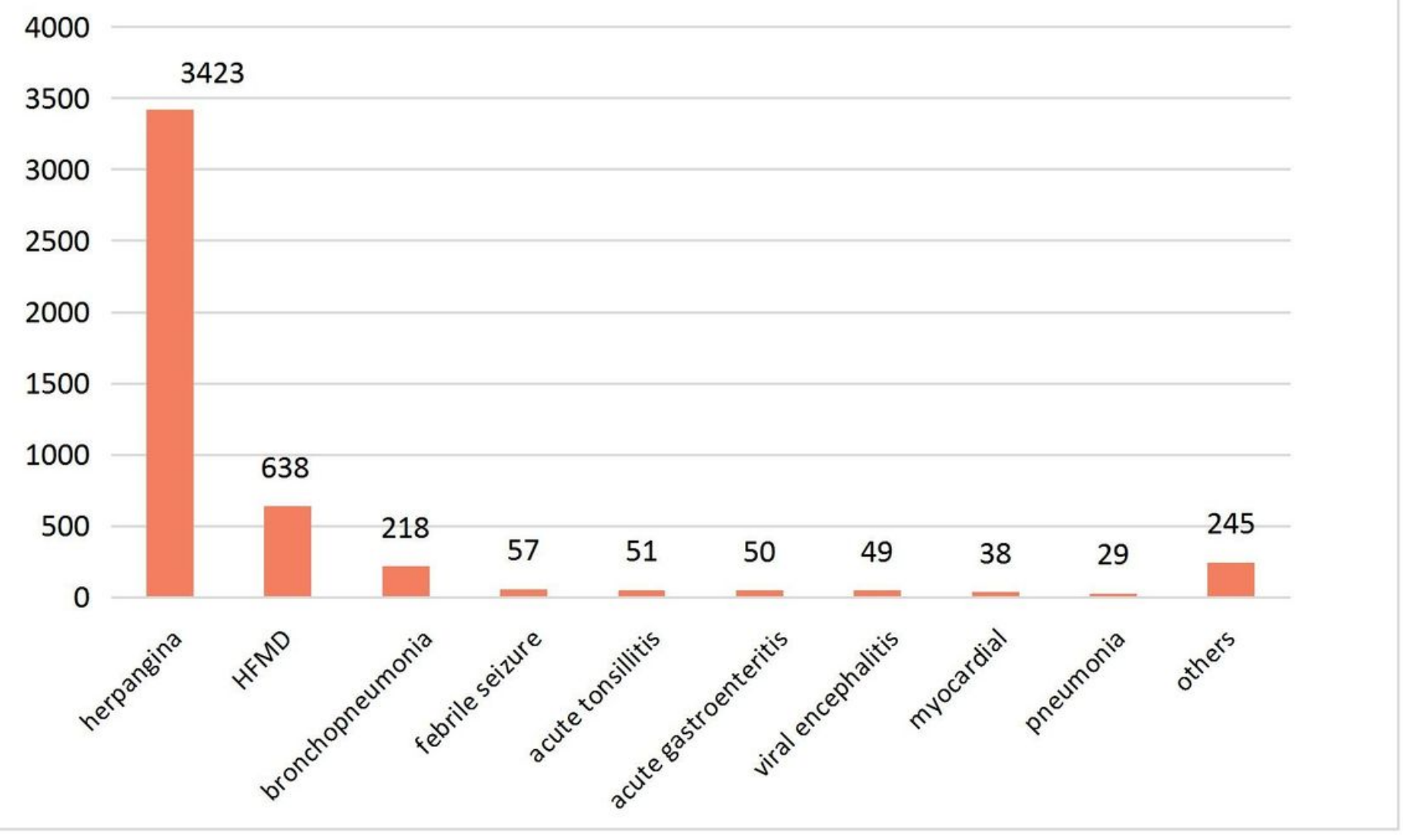

Figure 3

Main diagnosis of patients 


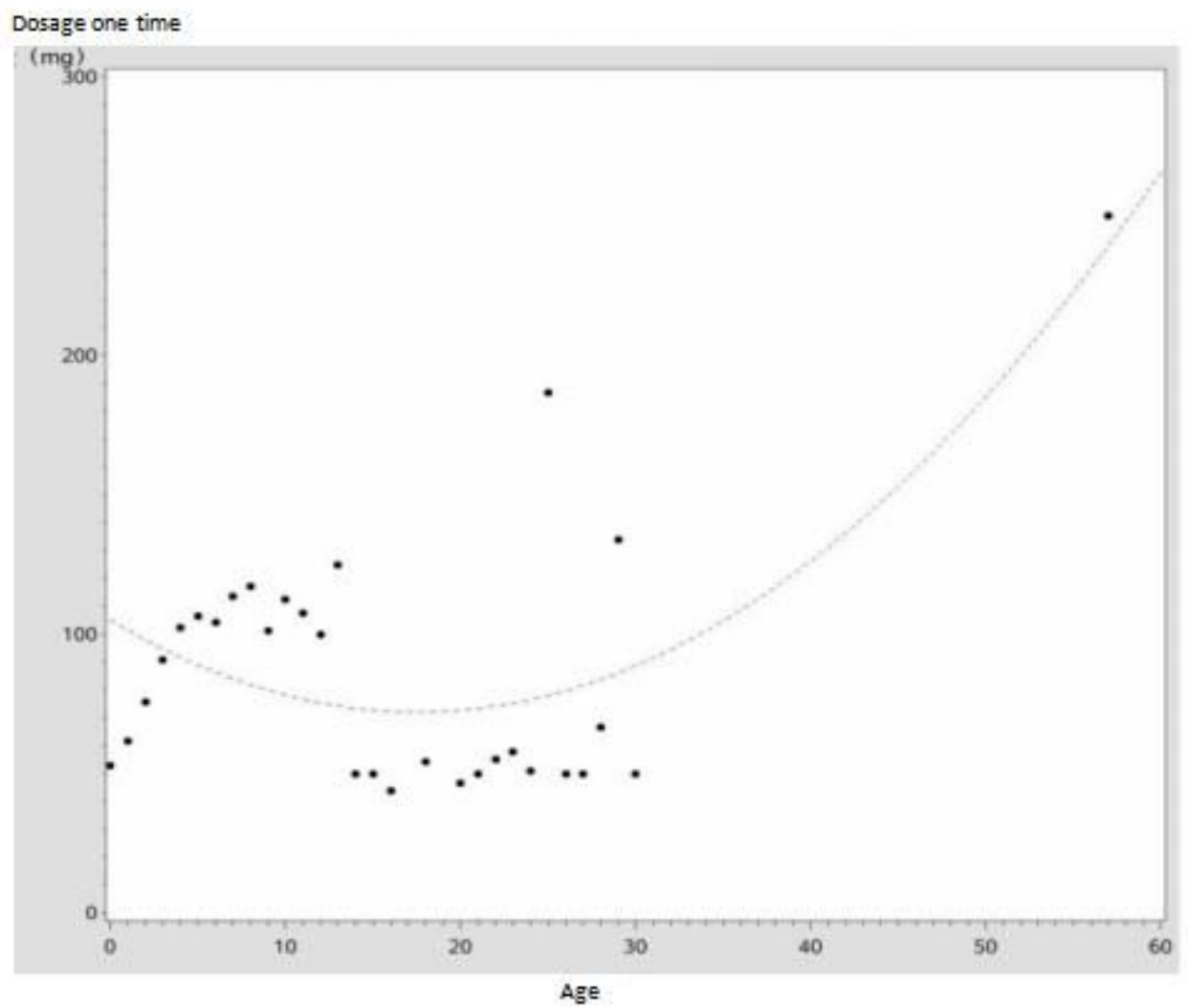

Figure 4

Regression relationship between age of patients and single doses

\section{Supplementary Files}

This is a list of supplementary files associated with this preprint. Click to download.

- Tables.docx 